\title{
Estimating the fractionally integrated process in the presence of measurement errors
}

\author{
Terence Tai-leung Chong*, Gilbert Chiu-sing Lui \\ Department of Economics, The Chinese University of Hong Kong, Shatin, Hong Kong \\ Received 29 October 1998; accepted 13 January 1999
}

\begin{abstract}
This paper studies the asymptotic bias of the differencing parameter estimator when data are measured with error. The estimator is established via the partial autocorrelation function. Factors that affect the direction of bias of the estimator are found. (c) 1999 Elsevier Science S.A. All rights reserved.
\end{abstract}

Keywords: Differencing parameter; Fractionally integrated model; Measurement error

JEL classification: C22

\section{Introduction}

Estimation of the fractional integrated model is currently a very active research area, see Baillie (1996) for an extensive review of the literature. In typical formulation of the model, it is usually assumed that the measured data conform to the variables of interest. Much less is known about the direction of bias of the estimate for the differencing parameter when errors in variables occur.

The consequences of errors in variables have been studied by a number of authors, one of the earliest was Adcock $(1877,1878)$, who considered the problem of fitting a straight line when both variables are subject to error. If only a single independent variable is measured with error, Levi (1973) showed that the corresponding OLS parameter estimate will be asymptotically biased towards zero. Nelson (1995) obtained a similar result for the case where more than one independent variable is measured with error. This phenomenon is called attenuation bias, a situation where the impact of the regressor on the dependent variable is diluted by measurement errors. The attenuation bias is usually linear, in the sense that the probability limit of the estimator is the true parameter multiplied by a positive constant less than 1 .

\footnotetext{
*Corresponding author.

E-mail address: b792703@mailserv.cuhk.edu.hk (T.T. Chong)
} 
This paper shows that the estimator for the differencing parameter in a fractionally integrated model is usually not attenuated when the data contain observational errors. Under some special situations, the estimator will be attenuated, but in a nonlinear fashion.

\section{Estimating d via partial autocorrelation function}

Consider the following model:

$$
(1-L)^{d} y_{t}^{*}=u_{t}, \quad t=1,2, \ldots, T,
$$

where $L$ is a lag operator such that $L y_{t}^{*}=y_{t-1}^{*}$.

The process $\left\{y_{t}^{*}\right\}$ is said to be integrated of order $d$ if $\left\{u_{t}\right\}$ is integrated of order zero. If $d$ is not an integer, then the process is said to be fractionally integrated (Granger and Joyeus, 1980). A fractionally integrated process is stationary but not invertible if $d \leq-0.5$. When $d \geq 0.5$, the process is nonstationary. In this paper, we assume for ease of exposition that the process under discussion is a fractionally integrated white noise process, with values of $d$ such that the process $y_{t}^{*}$ is stationary and invertible. Namely, we study cases where $u_{t} \sim$ i.i.d. $\left(0, \sigma_{u}^{2}\right), \sigma_{u}^{2}<\infty$ and $d \in(-0.5,0.5)$.

The fractional difference operator $(1-L)^{d}$ is given by

$$
(1-L)^{d}=\sum_{j=0}^{\infty} \frac{\Gamma(j-d)}{\Gamma(-d) \Gamma(j+1)} L^{j} .
$$

The expressions for autocorrelation and partial autocorrelation functions are especially simple when $u_{t}$ is assumed to be i.i.d. In particular, the $n^{\text {th }}$ order autocorrelation function of $y_{t}^{*}$ is provided by

$$
\rho_{n}(d)=\prod_{i=1}^{n} \frac{d+i-1}{i-d}
$$

while the $j^{\text {th }}$ order partial autocorrelation function is given by

$$
\alpha_{n}(d)=\frac{d}{n-d}
$$

A key problem in the fractionally integrated model is the determination of the degree of integration. In principle, we can estimate $d$ via $\rho_{n}(d)$ or $\alpha_{n}(d)$. We choose the latter one as it has a unique mapping with $d$, whereas $\rho_{n}(d)$ does not have this nice property.

To obtain the sample autocorrelation function, we run a regression of $y_{t}^{*}$ on $y_{t-1}^{*}, y_{t-2}^{*}, \ldots, y_{t-n}^{*}$. Let the estimated model be

$$
\hat{y}_{t}^{*}=\sum_{j=1}^{n} \hat{\beta}_{n, j} y_{t-j}^{*}
$$

As stated in Brockwell and Davis (1991, page 524), by the Durbin-Levinson algorithm (Brockwell and Davis, 1991, page 169, proposition 5.2.1), 


$$
\hat{\beta}_{n, j} \stackrel{p}{\rightarrow}-C_{j}^{n} \frac{\Gamma(j-d) \Gamma(n-d-j+1)}{\Gamma(-d) \Gamma(n-d+1)}, \quad j=1, \ldots, n
$$

In particular,

$$
\hat{\beta}_{n, n} \stackrel{p}{\rightarrow} \frac{d}{n-d}=\alpha_{n}(d)
$$

Thus, the $n^{\text {th }}$ order sample partial autocorrelation can be obtained from the estimated coefficient of $y_{t-n}^{*}$ in the regression of $y_{t}^{*}$ on $y_{t-1}^{*}, y_{t-2}^{*}, \ldots, y_{t-n}^{*}$.

The estimator for $d$ based on the $n$th order partial autocorrelation function can be constructed by

$$
\hat{d}(n)=\frac{n \hat{\beta}_{n, n}}{1+\hat{\beta}_{n, n}} .
$$

\section{Asymptotic bias of $\hat{d}(n)$}

Due to the presence of measurement errors, the true values of $\left\{y_{t}^{*}\right\}_{t=1}^{T}$ are not observable. Instead, we observe

$$
y_{t}=y_{t}^{*}+\varepsilon_{t}, \quad t=1,2, \ldots, T,
$$

where $\left\{\varepsilon_{t}\right\}_{t=1}^{T}$ is the measurement error process.

The case where $y_{t}^{*}$ follows an I(1) process (i.e., $d=1$ ) has been studied by Chong (1997). This paper will focus on the direction of bias of $\hat{d}(n)$ for $d \in(-0.5,0.5)$. To begin with, note that the $j$ th $(j=0,1,2, \ldots, T-1)$ order sample autocorrelation based on the observed data $\left\{y_{t}\right\}_{t=1}^{T}$ are unavoidably inconsistent as

$$
\hat{\rho}_{j}=\frac{\sum_{t=1}^{T-j}\left(y_{t}-\bar{y}\right)\left(y_{t+j}-\bar{y}\right)}{\sum_{t=1}^{T}\left(y_{t}-\bar{y}\right)^{2}} \stackrel{p}{\rightarrow} \frac{\sigma_{*}^{2} \rho_{j}+\gamma_{j}}{\sigma_{*}^{2}+\gamma_{0}}=A\left(\rho_{j}+\frac{\gamma_{j}}{\sigma_{*}^{2}}\right)
$$

where

$$
\begin{aligned}
& A=\frac{\sigma_{*}^{2}}{\sigma_{*}^{2}+\gamma_{0}}, \\
& \sigma_{*}^{2}=\operatorname{Var}\left(y_{t}^{*}\right)=\sigma_{u}^{2} \frac{\Gamma(1-2 d)}{\Gamma^{2}(1-d)}, \\
& \gamma_{j}=\operatorname{Cov}\left(\varepsilon_{t}, \varepsilon_{t+j}\right) .
\end{aligned}
$$


The direction of bias of $\hat{\rho}_{j}$ depends on the values of $A$ and the ratio $\frac{\gamma_{j}}{\sigma_{*}^{2}}$. In the case of uncorrelated measurement error (i.e., $\gamma_{j}=0$ for $j= \pm 1, \pm 2, \ldots$ ), $\hat{\rho}_{j}$ will be attenuated by a factor $A$.

To study the direction of bias of $\hat{d}(n)$ in (8), let

$$
\begin{aligned}
\hat{\boldsymbol{\beta}}(n) & =\left(\begin{array}{llll}
\hat{\beta}_{n, 1} & \hat{\beta}_{n, 2} \cdots \hat{\beta}_{n, n-1} & \hat{\beta}_{n, n}
\end{array}\right)^{\prime}, \\
\boldsymbol{\rho}(n) & =\left(\begin{array}{lllll}
\rho_{1} & \rho_{2} \cdots \rho_{n-1} & \rho_{n}
\end{array}\right)^{\prime}, \\
\boldsymbol{\gamma}(n) & =\left(\begin{array}{lllll}
\gamma_{1} & \gamma_{2} \cdots \gamma_{n-1} & \gamma_{n}
\end{array}\right)^{\prime}, \\
\boldsymbol{\Phi}(n) & =\left(\begin{array}{llll}
A^{-1} & \rho_{1}+\frac{\gamma_{1}}{\sigma_{*}^{2}} & \cdots & \rho_{n-1}+\frac{\gamma_{n-1}}{\sigma_{*}^{2}} \\
\rho_{1}+\frac{\gamma_{1}}{\sigma_{*}^{2}} & A^{-1} & \cdots & \rho_{n-2}+\frac{\gamma_{n-2}}{\sigma_{*}^{2}} \\
\vdots & \vdots & \ddots & \vdots \\
\rho_{n-1}+\frac{\gamma_{n-1}}{\sigma_{*}^{2}} & \rho_{n-2}+\frac{\gamma_{n-2}}{\sigma_{*}^{2}} & \cdots & A^{-1}
\end{array}\right) .
\end{aligned}
$$

Suppose we run a regression of $y_{t}$ on $y_{t-1}, y_{t-2}, \ldots, y_{t-n}$, the estimators converge in probability to:

$$
\hat{\boldsymbol{\beta}}(n) \stackrel{p}{\rightarrow} \boldsymbol{\Phi}(n)^{-1}\left(\boldsymbol{\rho}(n)+\sigma_{*}^{-2} \boldsymbol{\gamma}(n)\right) .
$$

\section{1. $\operatorname{ARMA}(1,1)$ measurement error}

For simplicity, we study the case where $\varepsilon_{t} \sim A R M A(1,1)$, which encompasses the pure $A R(1)$, pure $M A(1)$ and the white noise processes as special cases.

Let

$$
\varepsilon_{t}=\phi \varepsilon_{t-1}+\mu_{t}+\theta \mu_{t-1}
$$

where $\left\{\mu_{t}\right\}_{t=1}^{T}$ are i.i.d. $\left(0, \sigma_{\mu}^{2}\right)$ with $\sigma_{\mu}^{2}<\infty .\left\{\mu_{t}\right\}_{t=1}^{T}$ and $\left\{u_{t}\right\}_{t=1}^{T}$ are independent. Define

$$
f(n, d, a, \theta, \phi)=\operatorname{plim} \hat{d}(n)=n \frac{l(n) \boldsymbol{\Phi}(n)^{-1}\left(\boldsymbol{\rho}(n)+\sigma_{*}^{-2} \boldsymbol{\gamma}(n)\right)}{1+l(n) \boldsymbol{\Phi}(n)^{-1}\left(\boldsymbol{\rho}(n)+\sigma_{*}^{-2} \boldsymbol{\gamma}(n)\right)}
$$

where

$$
\begin{aligned}
& a=\frac{\sigma_{\mu}^{2}}{\sigma_{u}^{2}}, \\
& l(n)=\left(\begin{array}{lllll}
\underbrace{0}_{n \text { terms }} & &
\end{array}\right)
\end{aligned}
$$




$$
\begin{aligned}
& \gamma_{0}=\left(1+\theta^{2}+2 \phi \theta\right) \sigma_{\mu}^{2}, \\
& \gamma_{1}=\frac{(1+\phi \theta)(\phi+\theta)}{1+\theta^{2}+2 \phi \theta} \gamma_{0}, \\
& \gamma_{j}=\phi \gamma_{j-1} \quad j=2,3, \ldots, \\
& A=\frac{\Gamma(1-2 d)}{\Gamma(1-2 d)+a\left(1+\theta^{2}+2 \phi \theta\right) \Gamma^{2}(1-d)} .
\end{aligned}
$$

$f(n, d, a, \theta, \phi)$ is a compact generalization of the probability limit of the estimator $\hat{d}(n)$ for different values of $n, d, a, \theta$ and $\phi$ under $\operatorname{ARMA}(1,1)$ measurement errors. It is a tedious function especially for $n>3$ and for non-zero $\theta$ and $\phi$. To give a simple illustration, consider the case where the measurement error $\varepsilon_{t}$ is a white noise such that $\theta=\phi=0, \gamma_{j}=0(j=1,2, \ldots)$. The corresponding functions for $n=1,2$ and 3 would be respectively

$$
\begin{aligned}
& f(1, d, a, 0,0)=\frac{\Gamma(1-2 d)}{(1-d) a \Gamma^{2}(1-d)+\Gamma(1-2 d)} d \\
& f(2, d, a, 0,0)=\frac{2 d \Gamma(1-2 d)\left(a\left(d^{2}-1\right) \Gamma^{2}(1-d)+(2 d-1) \Gamma(1-2 d)\right)}{H_{0}}, \\
& f(3, d, a, 0,0)=\frac{-3 d \Gamma(1-2 d) H_{1}}{\left(a(d-1) \Gamma^{2}(1-d)+(2 d-1) \Gamma(1-2 d)\right) H_{2}},
\end{aligned}
$$

where

$$
\begin{aligned}
H_{0}= & a^{2}(d-2)(d-1)^{2} \Gamma^{4}(1-d)+2(2 d-1) \Gamma^{2}(1-2 d) \\
& +a(d-1)\left(3 d^{2}-5 d+4\right) \Gamma(1-2 d) \Gamma^{2}(1-d), \\
H_{1}= & a^{2}(d+1)\left(d^{2}-4\right)(d-1)^{2} \Gamma^{4}(1-d)-4(2 d-1)^{2} \Gamma^{2}(1-2 d) \\
& +4 a(d+1)(d-2)(2 d-1)(d-1) \Gamma(1-2 d) \Gamma^{2}(1-d), \\
H_{2}= & a^{2}(d-3)(d-2)^{2}(d-1)^{2} \Gamma^{4}(1-d)+12(2 d-1) \Gamma^{2}(1-2 d) \\
& -2 a(d-1)(d-2)\left(5 d^{2}-7 d+6\right) \Gamma(1-2 d) \Gamma^{2}(1-d) .
\end{aligned}
$$

Fig. 1 plots the shape of $f(1, d, a, 0,0)$ for $a=1,5$ and 10.

Observe from Fig. 1 that

$$
|f(1, d, a, 0,0)| \leq|d|
$$

and

$$
\frac{\partial}{\partial a}|f(1, d, a, 0,0)| \leq 0 .
$$




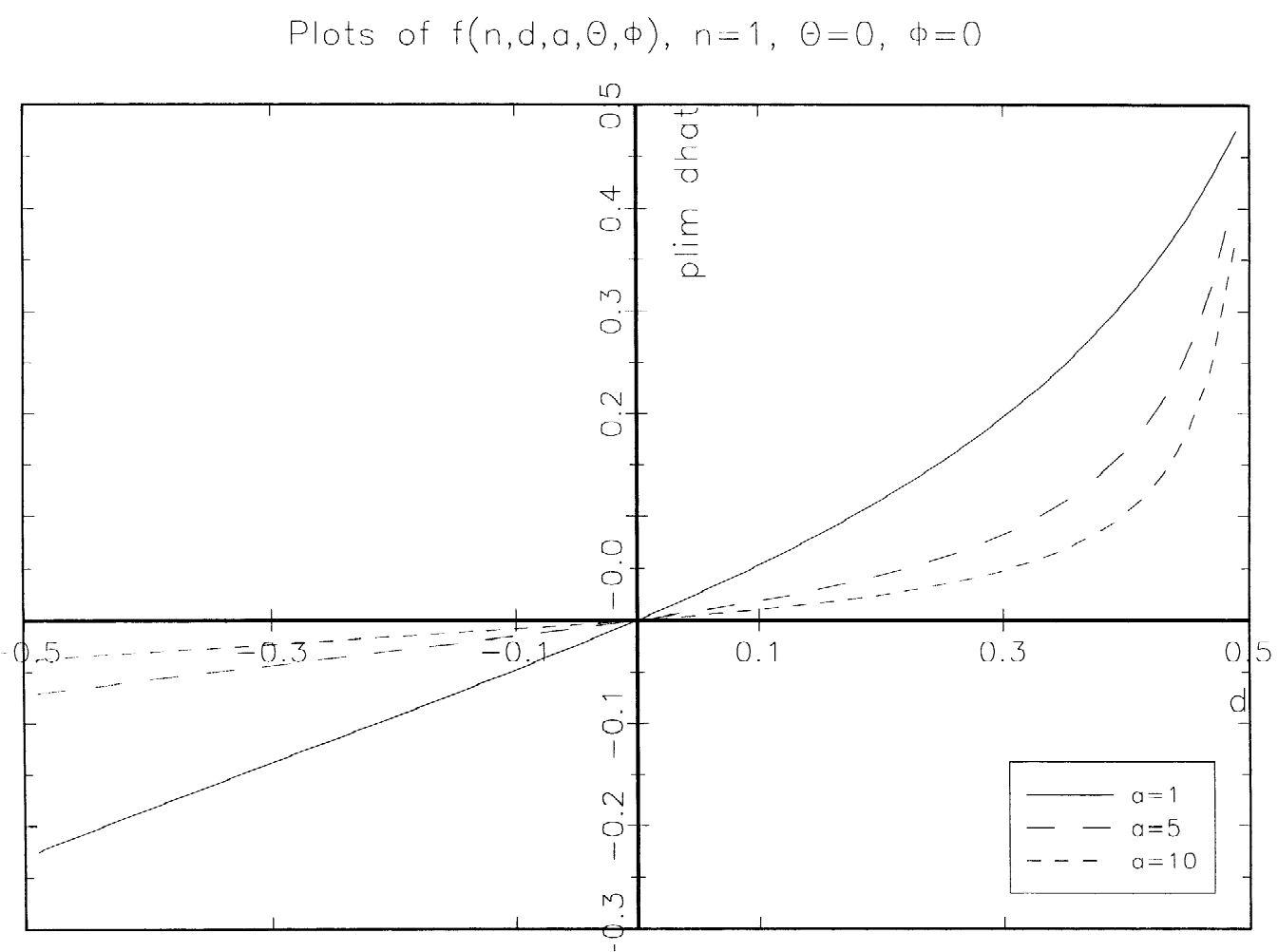

Fig. 1. Plots of $f(n, d, a, \theta, \phi), n=1, \theta=0, \phi=0$.

Thus for white noise measurement errors and for $n=1$, the estimator for $d$ is biased towards zero. As the variance of measurement error increases (the value of $a$ increases), the estimator will bias more towards zero.

Fig. 2 shows the effect of $n$ on $f(n, d, a, 0,0)$, using $a=1$ and $n=1,4$.

Note from Fig. 2 that $f(1, d, 1,0,0) \leq f(4, d, 1,0,0)$. The equality holds only when $d=0$. Generally

Table 1

Numerical values of $f(1, d, 1, \theta, \phi)$

\begin{tabular}{llllllll}
\hline$d \backslash(\theta, \phi)$ & $(-0.5,-0.5)$ & $(0,-0.5)$ & $(-0.5,0)$ & $(0,0)$ & $(0.5,0)$ & $(0,0.5)$ & $(0.5,0.5)$ \\
\hline-0.49 & -1.3747 & -0.7145 & -0.5729 & -0.2248 & 0.0325 & 0.0882 & 0.2581 \\
-0.4 & -1.3261 & -0.6646 & -0.5254 & -0.1832 & 0.0624 & 0.1155 & 0.2742 \\
-0.3 & -1.2649 & -0.6070 & -0.4715 & -0.1381 & 0.0937 & 0.1439 & 0.2907 \\
-0.2 & -1.1933 & -0.5455 & -0.4151 & -0.0935 & 0.1235 & 0.1707 & 0.3058 \\
-0.1 & -1.1070 & -0.4777 & -0.3541 & -0.0480 & 0.1526 & 0.1966 & 0.3199 \\
0 & -1.0000 & -0.4000 & -0.2857 & 0 & 0.1818 & 0.2222 & 0.3333 \\
0.1 & -0.8633 & -0.3075 & -0.2054 & 0.0531 & 0.2127 & 0.2490 & 0.3468 \\
0.2 & -0.6823 & -0.1922 & -0.1061 & 0.1157 & 0.2480 & 0.2791 & 0.3613 \\
0.3 & -0.4328 & -0.0402 & 0.0244 & 0.1959 & 0.2931 & 0.3172 & 0.3794 \\
0.4 & -0.0696 & 0.1733 & 0.2095 & 0.3101 & 0.3615 & 0.3755 & 0.4089 \\
0.49 & 0.4291 & 0.4597 & 0.4636 & 0.4752 & 0.4794 & 0.4808 & 0.4819 \\
\hline
\end{tabular}




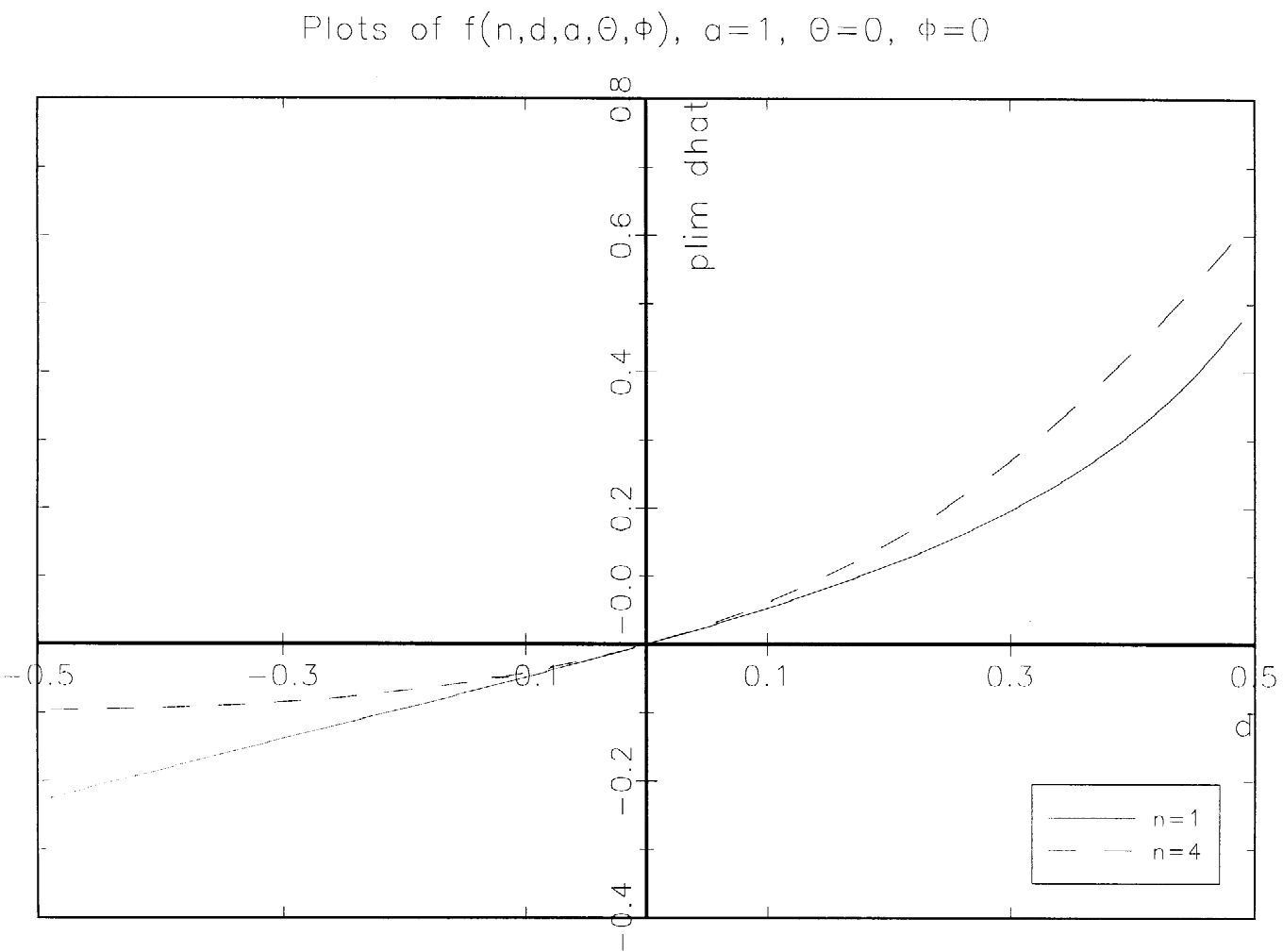

Fig. 2. Plots of $f(n, d, a, \theta, \phi), a=1, \theta=0, \phi=0$.

speaking, $f(1, d, a, 0,0)$ serves as a lower support for $f(n, d, a, 0,0)$. Thus, for $d>0$, using a higher order partial autocorrelation may improve the estimate of $d$.

Using the formula presented in $(20), f(n, d, a, \theta, \phi)$ is easily evaluated on a computer. A Gauss program that calculates the values of $f(n, d, a, \theta, \phi)$ is available from us. Tables 1 to 3 report the numerical values of $f(n, d, a, \theta, \phi)$ for $n=1,2$ and $3, \theta=0, \pm 0.5$ and $\phi=0, \pm 0.5$. The tables give a

Table 2

Numerical values of $f(2, d, 1, \theta, \phi)$

\begin{tabular}{llllllll}
\hline$d \wedge(\theta, \phi)$ & $(-0.5,-0.5)$ & $(0,-0.5)$ & $(-0.5,0)$ & $(0,0)$ & $(0.5,0)$ & $(0,0.5)$ & $(0.5,0.5)$ \\
\hline-0.49 & -0.4726 & -0.2087 & -0.4753 & -0.1593 & -0.0726 & 0.1602 & 0.1802 \\
-0.4 & -0.3981 & -0.1552 & -0.4213 & -0.1373 & -0.0819 & 0.1537 & 0.1514 \\
-0.3 & -0.3105 & -0.0930 & -0.3563 & -0.1107 & -0.0925 & 0.1456 & 0.1166 \\
-0.2 & -0.2163 & -0.0268 & -0.2843 & -0.0804 & -0.1020 & 0.1373 & 0.0793 \\
-0.1 & -0.1136 & 0.0451 & -0.2030 & -0.0445 & -0.1087 & 0.1299 & 0.0401 \\
0 & 0 & 0.1250 & -0.1096 & 0 & -0.1096 & 0.1250 & 0 \\
0.1 & 0.1275 & 0.2157 & -0.0003 & 0.0572 & -0.1000 & 0.1256 & -0.0390 \\
0.2 & 0.2723 & 0.3212 & 0.1298 & 0.1332 & -0.0717 & 0.1370 & -0.0725 \\
0.3 & 0.4386 & 0.4467 & 0.2871 & 0.2378 & -0.0093 & 0.1700 & -0.0903 \\
0.4 & 0.6313 & 0.5990 & 0.4798 & 0.3867 & 0.1192 & 0.2498 & -0.0603 \\
0.49 & 0.8311 & 0.7667 & 0.6920 & 0.5802 & 0.3532 & 0.4201 & 0.1297 \\
\hline
\end{tabular}


Table 3

Numerical values of $f(3, d, 1, \theta, \phi)$

\begin{tabular}{llllllll}
\hline$d \wedge(\theta, \phi)$ & $(-0.5,-0.5)$ & $(0,-0.5)$ & $(-0.5,0)$ & $(0,0)$ & $(0.5,0)$ & $(0,0.5)$ & $(0.5,0.5)$ \\
\hline-0.49 & -0.5114 & -0.2986 & -0.4047 & -0.1209 & -0.0374 & 0.0978 & 0.0204 \\
-0.4 & -0.4336 & -0.2675 & -0.3545 & -0.1105 & -0.0346 & 0.0828 & 0.0083 \\
-0.3 & -0.3397 & -0.2271 & -0.2915 & -0.0948 & -0.0272 & 0.0680 & -0.0013 \\
-0.2 & -0.2369 & -0.1786 & -0.2191 & -0.0730 & -0.0143 & 0.0559 & -0.0064 \\
-0.1 & -0.1241 & -0.1197 & -0.1350 & -0.0425 & 0.0059 & 0.0479 & -0.0063 \\
0 & 0 & -0.0476 & -0.0370 & 0 & 0.0361 & 0.0462 & 0 \\
0.1 & 0.1362 & 0.0483 & 0.0777 & 0.0593 & 0.0808 & 0.0544 & 0.0147 \\
0.2 & 0.2853 & 0.1493 & 0.2117 & 0.1421 & 0.1476 & 0.0797 & 0.0424 \\
0.3 & 0.4473 & 0.2816 & 0.3673 & 0.2571 & 0.2494 & 0.1357 & 0.0944 \\
0.4 & 0.6213 & 0.4409 & 0.5456 & 0.4154 & 0.4094 & 0.2516 & 0.2037 \\
0.49 & 0.7866 & 0.6087 & 0.7248 & 0.6053 & 0.6368 & 0.4615 & 0.4573 \\
\hline
\end{tabular}

feel for how the direction and magnitude of bias change with these parameters. Cases where $(\theta$, $\phi)=(-0.5,0.5)$ and $(0.5,-0.5)$ are equivalent to the white noise case and are therefore skipped. Some features in Table 1 deserve attention:

i) Attenuation bias occurs only in the case where $(\theta, \phi)=(0,0)$.

ii) For $\phi_{a}<\phi_{b}$, and for any $\theta_{a}, \theta_{b} \in(-1,1), f\left(1, d, 1, \theta_{a}, \phi_{a}\right) \leq f\left(1, d, 1, \theta_{b}, \phi_{b}\right)$. This implies the

Plots of $f(n, d, a, \Theta, \phi), n-1, a=1$

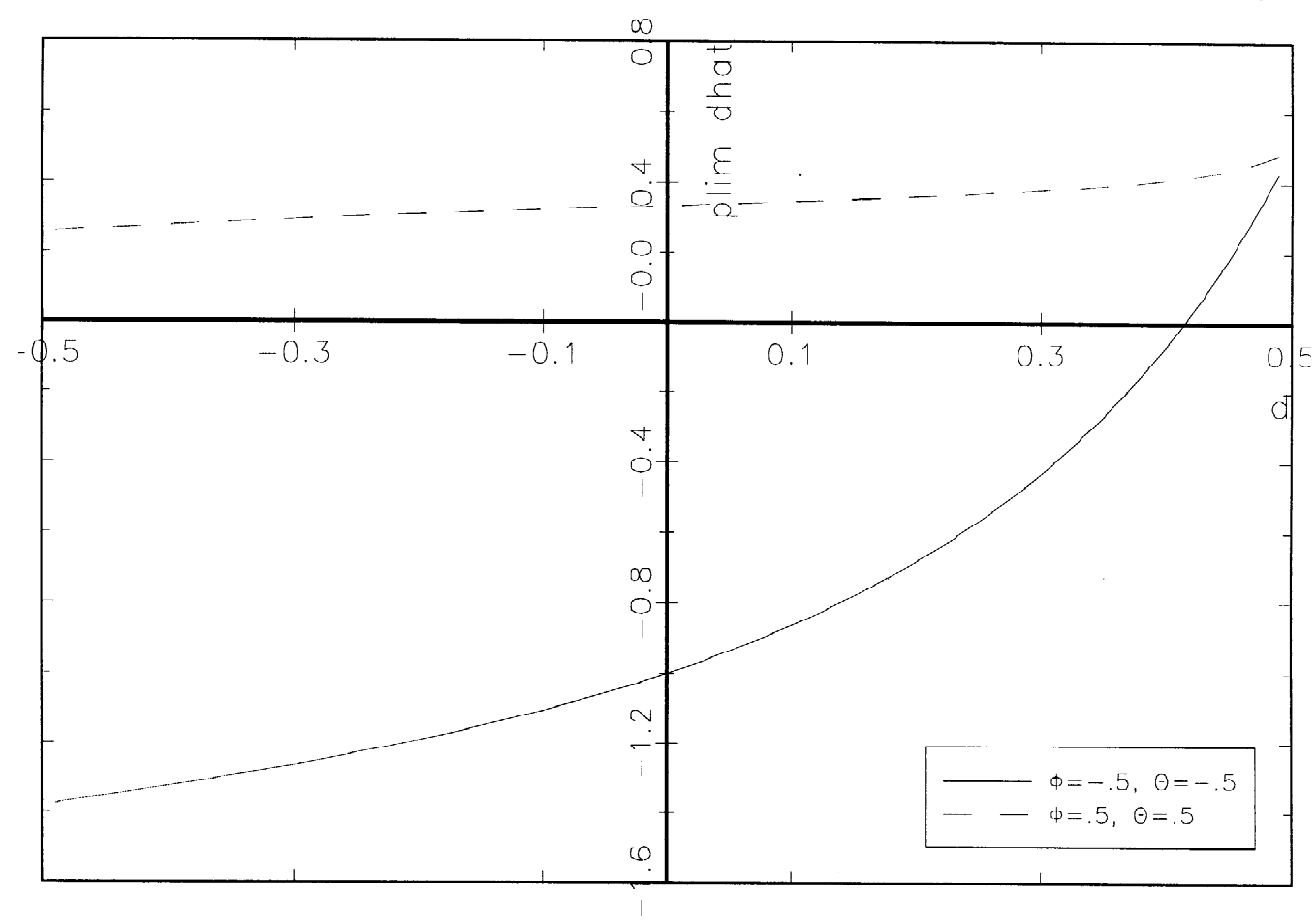

Fig. 3. Plots of $f(n, d, a, \theta, \phi), n=1, a=1$. 
autoregressive parameter $\phi$ plays a dominant role in deciding the direction of bias of $\hat{d}(n)$. Note that $f(1, d, 1, \theta, \phi)>0$ for $(\theta, \phi)=(0.5,0),(0,0.5),(0.5,0.5)$. Thus no matter the memory of the true process is long or short, it will likely be identified as a long-memory process under these kinds of measurement errors. On the contrary, for $(\theta, \phi)=(-0.5,-0.5)$, it will likely be identified as a short-memory process. Graphs of $f(1, d, 1,0.5,0.5)$ and $f(1, d, 1,-0.5,-0.5)$ are shown in Fig. 3.

iii) For $\theta_{a}<\theta_{b}, f\left(1, d, 1, \theta_{a}, \phi\right) \leq f\left(1, d, 1, \theta_{b}, \phi\right)$, i.e., given the value of the $\phi$, a higher value of the moving average parameter yields a higher value of $p \lim \hat{d}(n)$.

iv) For $d_{1}<d_{2}, f\left(1, d_{1}, 1, \theta, \phi\right) \leq f\left(1, d_{2}, 1, \theta, \phi\right)$. Thus $f(1, d, 1, \theta, \phi)$ is monotonically increasing with $d$. That means the higher the true value of $d$, the larger the probability limit of the estimator under measurement errors. This appears to be a reasonable outcome. Interestingly, Tables 2 and 3 suggest the feature may be deprived in cases where $n>1$.

Note that $f(2, d, 1,0.5,0), f(2, d, 1,0,0.5), f(2, d, 1,-0.5,0.5), f(3, d, 1,0,0.5)$ and $f(3, d, 1,0.5$, $0.5)$ are all non-monotonic in $d$.

Observe from Tables 1 to 3 that the values of $f(n, d, a, \theta, \phi)$ increase dramatically when $d$ approaches 0.5 . This is because as $d$ closes to 0.5 , the true process $y_{t}^{*}$ will be nearly nonstationary and becomes more volatile. The stationary measurement error process $\varepsilon_{t}$ is therefore dominated by $y_{t}^{*}$, and the estimate of $d$ based on the observed process $y_{t}$ are generally larger than 0.5 .

A totally different conclusion on the degree of memory and the stationarity of the original process can be produced by the existence of measurement errors. A surprising case is depicted in Fig. 4 where $n=2$ and $(\theta, \phi)=(0.5,0.5)$.

$$
\text { Plots of } f(n, d, a, \Theta, \phi), n=2, a=1
$$

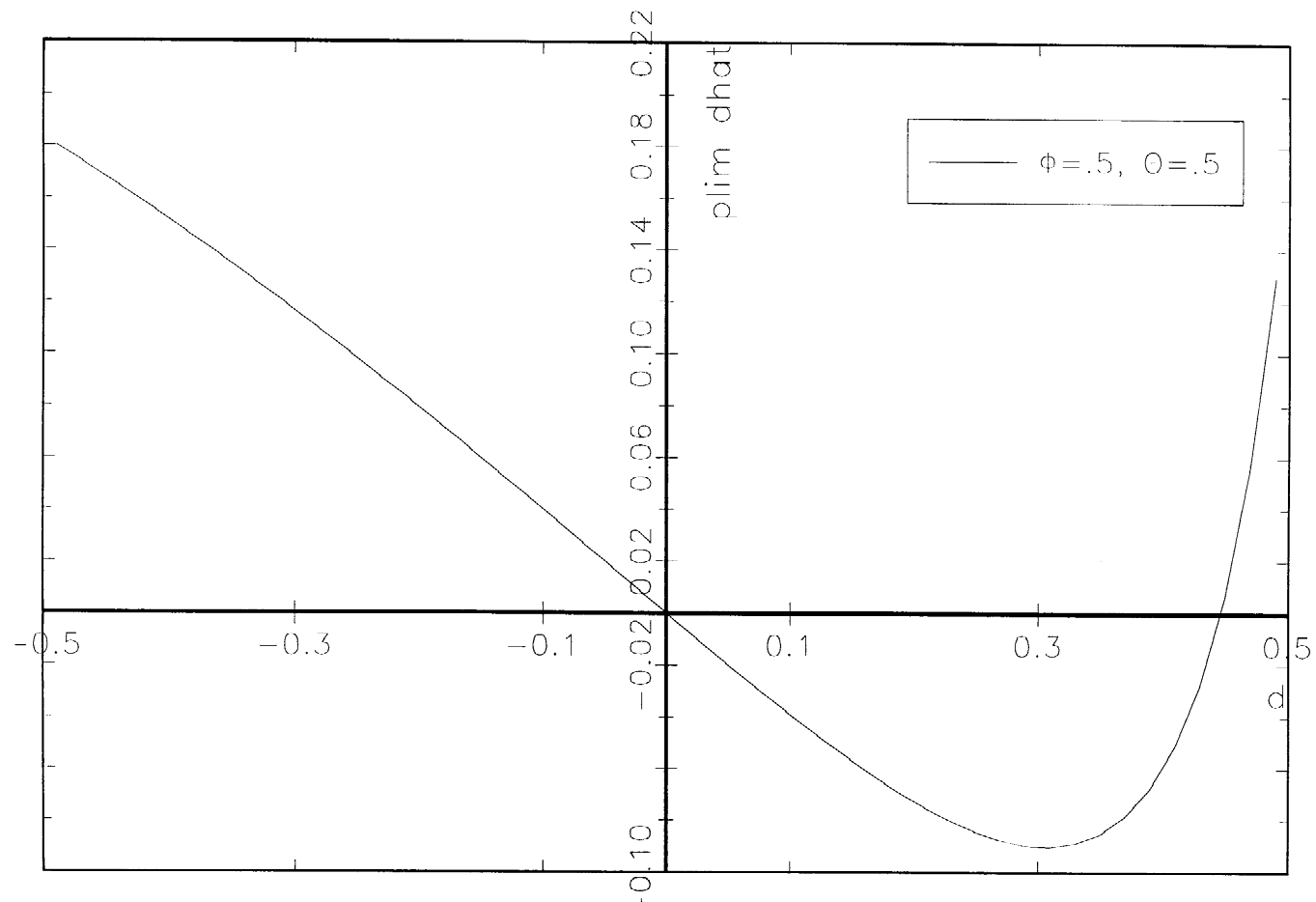

Fig. 4. Plots of $f(n, d, a, \theta, \phi), n=2, a=1$. 
Note that the sign of $f(2, d, 1,0.5,0.5)$ is opposite to that of $d$, except for $d$ near 0.5 . A short-memory process $(d<0)$ with this kind of measurement error will be wrongly identified as a long-memory one, and vice versa. When the process is nearly nonstationary $(d=0.49)$, the true value of $d$ will be substantially underestimated $(f(2,0.49,1,0.5,0.5)=0.1297)$, thus a nonstationary process may be wrongly reported as a stationary one.

\section{Further developments}

This paper shows that the direction of bias of differencing parameter estimate of a fractionally integrated model is drastically affected by measurement error. We obtain the conditions under which the estimator is asymptotically biased towards zero. As a starting point, we assume that the mean of the original process $y_{t}^{*}$ is zero and the innovations $u_{t}$ are i.i.d.. Priorities for future research include: allowing for the mean of $y_{t}^{*}$ to be non-zero; allowing for a non-i.i.d. innovation $u_{t}$; using a different estimator for $d$; and allowing for ARFIMA measurement errors.

\section{References}

Adcock, R.J., 1877. Note on the method of least squares. The Analyst 4, 183-184.

Adcock, R.J., 1878. A problem in least squares. The Analyst 4, 53-54.

Baillie, R.T., 1996. Long memory processes and fractional integration in econometrics. Journal of Econometrics 73, 5-59. Brockwell, P.J., Davis, R.A., 1991. Time Series: Theory and Methods, 2nd edn, Springer-Verlag.

Chong, T.L., 1997. Estimating the unit root process in the presence of measurement errors. Journal of Applied Statistical Science $6(2 / 3), 105-120$.

Granger, C.W.J., Joyeus, R., 1980. An introduction to the long-memory time series models and fractional differencing. Journal of Time Series Analysis 1, 15-29.

Levi, M.D., 1973. Errors in the variables bias in the presence of correctly measured variables. Econometrica 41, $985-986$. Nelson, D.B., 1995. Vector attenuation bias in the classical errors-in-variables model. Economics Letters 49, $345-349$. 